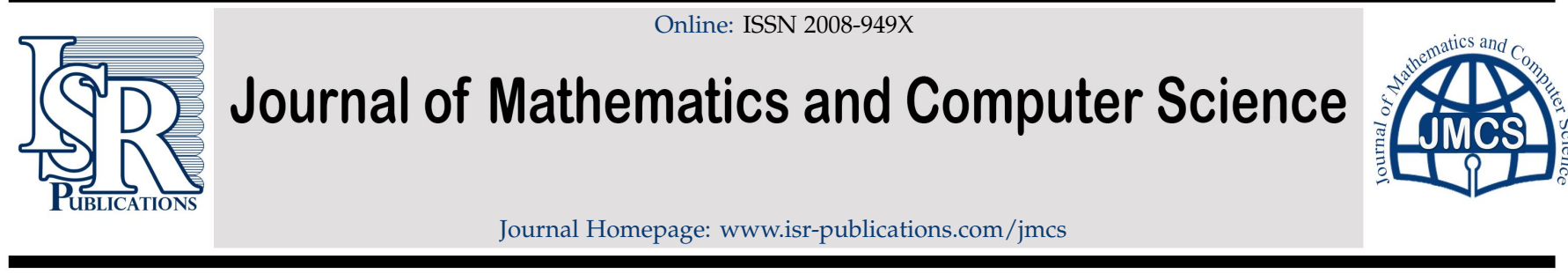

\title{
Statistical convergence in non-archimedean Köthe sequence spaces
}

\author{
D. Eunice Jemima ${ }^{a, *}$, V. Srinivasan ${ }^{b}$
}

${ }^{a}$ Department of Mathematics, Faculty of Engineering and Technology, SRM Institute of Science and Technology, Kattankulathur, Chennai-603203, India.

${ }^{b}$ (Retd. Professor) Department of Mathematics, Faculty of Engineering and Technology, SRM Institute of Science and Technology, Kattankulathur, Chennai-603203, India.

\begin{abstract}
The aim of this paper is to examine statistical convergence in a Köthe sequence space, when the sequences have their entries in a non-archimedean field $\mathscr{K}$ which is both non-trivial and complete under the metric induced by the valuation $||:. \mathscr{K} \rightarrow[0, \infty)$, which is denoted by $K(B)$.
\end{abstract}

Keywords: Köthe space, non-archimedean field, non-archimedean Köthe space, statistical convergence.

2020 MSC: 40A35, 46E30, 46S10.

(C)2021 All rights reserved.

\section{Introduction}

In classical analysis, the study of certain pairs of subspaces of the space of all real sequences was initiated by Köthe and Toeplitz, and a little later by Köthe alone. Lorentz and Wertheim, Dieudonne and Cooper generalized their concept.

A set $\mathcal{A}$ of non-negative sequences $\left(\alpha_{n}\right)_{n \in N}$ is called a Köthe set, if

(i) for each $n \in N$, there exists $\alpha \in \mathcal{A}$ with $\alpha_{n}>0$;

(ii) for each pair $(\alpha, \beta) \in \mathcal{A} \times \mathcal{A}$, there exists a $\gamma \in \mathcal{A}$ such that $\max \left(\alpha_{n}, \beta_{n}\right) \leqslant \gamma_{n}$, for all $n \in N$.

Köthe sequence spaces are defined classically as the orthogonals of certain subsets of Köthe sets. They are locally convex topological vector spaces which are Hausdorff and complete.

\subsection{Non-archimedean Köthe spaces}

Definition 1.1. Consider an infinite matrix $B=\left(b_{n, k}\right)$ consisting of positive real numbers, and satisfying the condition

$$
b_{n, k} \leqslant b_{n, k+1}, \quad n, k=1,2, \cdots .
$$

The non-archimedean Köthe space K(B) associated with the matrix B is defined by De Grande-De Kimpe

\footnotetext{
*Corresponding author

Email addresses: eunicejem@gmail .com (D. Eunice Jemima), drvsrinivas.5@gmail . com (V. Srinivasan)

doi: $10.22436 /$ jmcs.023.02.01
}

Received: 2020-07-22 Revised: 2020-08-04 Accepted: 2020-08-17 
[3] and Sliwa [11] as

$$
\mathrm{K}(\mathrm{B})=\left\{\left(\alpha_{n}\right), \alpha_{n} \in \mathscr{K}:\left|\alpha_{n}\right| b_{n, k} \rightarrow 0, \quad n, k=1,2, \cdots\right\},
$$

with the sequence of norms

$$
\left|\left(\alpha_{n}\right)\right|_{k}=\max _{n}\left|\alpha_{n}\right| b_{n, k}, \quad k=1,2, \cdots .
$$

Example 1.2. Consider the matrix $B=\left(b_{n, k}\right)$ where

$$
b_{n, k}=\exp \left(-b_{n} / k\right),
$$

and $\left(b_{n}\right)$ is a non-decreasing sequence of positive real numbers with $\lim _{n \rightarrow \infty} b_{n}=\infty$. Then $K(B)$ is a Köthe space associated with the matrix B since,

$$
\begin{aligned}
|| \alpha_{n}\left|b_{n, k}\right| & =\max _{n}|| \alpha_{n}\left|b_{n, k}\right| \\
& =\max _{n}\left|\alpha_{n}\right| e^{-b_{n} / k} \\
& =\max _{n}\left|\alpha_{n}\right| \frac{1}{e^{b_{n} / k}} \rightarrow 0, \text { as } n \rightarrow \infty .
\end{aligned}
$$

That is, $\lim _{n \rightarrow \infty}|| \alpha_{n}\left|b_{n, k}\right|=0$.

Definition 1.3. Let $\mathscr{K}$ be a complete, non-trivially valued, non-archimedean field. In [12], a sequence $x=\left(x_{k}\right), x_{k} \in \mathscr{K}, k=1,2, \cdots$, is defined to be statistically convergent to a limit $l$, if for every $\epsilon>0$,

$$
\lim _{n \rightarrow \infty} \frac{1}{n}\left|\left\{k \leqslant n:\left|x_{k}-l\right| \geqslant \epsilon\right\}\right|=0,
$$

where the outer vertical bars stand for the set's cardinality. Symbolically, it is written as stat-lim $x_{k}=l$ or $x_{\mathrm{k}} \stackrel{\text { stat }}{\longrightarrow} l$.

We now define statistical convergence in a non-archimedean Köthe space as follows.

Definition 1.4. A sequence $x=\left(x_{n}\right)$ of a non-archimedean Köthe space $K(B)$ is said to be statistically convergent to a limit $l$, if for any $\epsilon>0$,

$$
\lim _{m \rightarrow \infty} \frac{1}{m}\left|\left\{n, k \leqslant m:|| x_{n}\left|b_{n, k}-l\right| \geqslant \epsilon\right\}\right|=0 .
$$

Symbolically, we write $x_{k} \rightarrow l\{S(K(B))\}$, where $\{S(K(B))\}$ denotes the set of statistically convergent non-archimedean Köthe spaces.

Definition 1.5. A sequence $x=\left(x_{n}\right)$ of a non-archimedean Köthe space $K(B)$ is said to be a statistically null sequence, if for any $\epsilon>0$,

$$
\lim _{m \rightarrow \infty} \frac{1}{m}\left|\left\{n, k \leqslant m:|| x_{n}\left|b_{n, k}\right| \geqslant \epsilon\right\}\right|=0 .
$$

\section{Main results}

Theorem 2.1. If a sequence $\mathrm{x}=\left(\mathrm{x}_{\mathrm{n}}\right)$ of a non-archimedean Köthe space $\mathrm{K}(\mathrm{B})$ is convergent to $\mathrm{l}$, then $\left(\mathrm{x}_{\mathrm{n}}\right)$ is statistically convergent to l. i.e., if $x_{n} \rightarrow l(K(B))$, then $x_{n} \rightarrow l\{S(K(B))\}$. 
Proof. Let us assume that a non-archimedean Köthe sequence $x=\left(x_{n}\right)$ is convergent to $l$. Then,

$$
\lim _{n \rightarrow \infty}|| x_{n}\left|b_{n, k}-l\right|=0
$$

which implies that,

$$
\lim _{n \rightarrow \infty} \max \left(\left|x_{n}\right| b_{n, k}-l\right)=0
$$

To prove $x_{n} \rightarrow l\{S(K(B))\}$, i.e., to prove $\lim _{m \rightarrow \infty} \frac{1}{m}\left|\left\{n, k \leqslant m:|| x_{n}\left|b_{n, k}-l\right| \geqslant \epsilon\right\}\right|=0$, consider

$$
\begin{aligned}
\frac{1}{m} \mid\{n, k \leqslant m & \left.:|| x_{n}\left|b_{n, k}-l\right| \geqslant \epsilon\right\} \mid \\
& =\frac{1}{m}\left|\left\{n, k \leqslant m: \max \left(\left|x_{n}\right| b_{n, k}-l\right) \geqslant \epsilon\right\}\right| \rightarrow 0 \text { as } m \rightarrow \infty, \quad \text { (using (2.1)). }
\end{aligned}
$$

Therefore, $\lim _{m \rightarrow \infty} \frac{1}{m}\left|\left\{n, k \leqslant m:|| x_{n}\left|b_{n, k}-l\right| \geqslant \epsilon\right\}\right|=0$. This implies that, $x_{n} \rightarrow l\{S(K(B))\}$.

Theorem 2.2. If a sequence $x=\left(x_{n}\right)$ of a non-archimedean Köthe space $\mathrm{K}(\mathrm{B})$ is statistically convergent to $\mathrm{l}$, then $\left(x_{n}\right)$ is convergent to l provided, sup ||$x_{n}\left|b_{n, k}\right|<\infty$. That is, $x_{n} \rightarrow l\{S(K(B))\}$ implies $x_{n} \rightarrow l(K(B))$ if $\sup _{n}|| x_{n}\left|b_{n, k}\right|<\infty$.

Proof. Let us assume that $\sup _{n}|| x_{n}\left|b_{n, k}\right|<\infty$. Then, there exists a positive integer $M>0$ such that,

$$
|| x_{n}\left|b_{n, k}\right| \leqslant M
$$

That is,

$$
|| x_{n}\left|b_{n, k}-l+l\right| \leqslant M
$$

which implies that

$$
\max \left(|| x_{n}\left|b_{n, k}-l\right|,|l|\right) \leqslant M .
$$

Also, since the Köthe sequence $\left(x_{n}\right)$ is statistically convergent to $l$, we have

$$
\lim _{m \rightarrow \infty} \frac{1}{m}\left|\left\{n, k \leqslant m:|| x_{n}\left|b_{n, k}-l\right| \geqslant \frac{\epsilon}{2}\right\}\right|<\frac{\epsilon}{2 M} .
$$

Let $I_{m}=\left\{n, k \leqslant m:|| x_{n}\left|b_{n, k}-l\right| \geqslant \frac{\epsilon}{2}\right\}$. Now, consider

$$
\begin{aligned}
\frac{1}{m} \sum_{n, k=1}^{m}|| x_{n}\left|b_{n, k}-l\right| & =\frac{1}{m} \sum_{n, k \in I_{m}}|| x_{n}\left|b_{n, k}-l\right|+\frac{1}{m} \sum_{n, k \notin I_{m}}|| x_{n}\left|b_{n, k}-l\right| \\
& \leqslant \frac{1}{m} \sup _{n, k \in I_{m}}|| x_{n}\left|b_{n, k}-l\right|+\frac{1}{m} \sup _{n, k \notin I_{m}}|| x_{n}\left|b_{n, k}-l\right| \\
& <\frac{1}{m}\left(m \cdot \frac{\epsilon}{2 M}\right) M+\frac{1}{m}\left(m \cdot \frac{\epsilon}{2}\right) \quad(\text { using (2.2) and (2.3)) } \\
& <\epsilon .
\end{aligned}
$$

Therefore, $\lim _{m \rightarrow \infty} \frac{1}{m} \sum_{n, k=1}^{m}|| x_{n}\left|b_{n, k}-l\right|=0$. That is, $\left(x_{n}\right)$ is convergent to $l$. 
Theorem 2.3. A sequence $\mathrm{x}=\left(\mathrm{x}_{\mathrm{n}}\right)$ of a non-archimedean Köthe space $\mathrm{K}(\mathrm{B})$ is statistically convergent to $\mathrm{l}$, if and only if the following condition is satisfied

$$
\lim _{m \rightarrow \infty} \frac{1}{m}\left|\left\{n, k, n^{\prime} \leqslant m:|| x_{n}\left|b_{n, k}-\right| x_{n^{\prime}(r)}\left|b_{n^{\prime}(r), k}\right| \geqslant \epsilon\right\}\right|=0,
$$

where $\left(x_{n^{\prime}(r)}\right)$ is a subsequence of $\left(x_{n}\right)$ such that $\left(x_{n^{\prime}(r)}\right)$ is convergent to $l$.

Proof. Let us assume that $\left(x_{n}\right)$ of a non-archimedean Köthe space is statistically convergent to $l$. Then, for any $\epsilon>0$,

$$
\lim _{m \rightarrow \infty} \frac{1}{m}\left|\left\{n, k \leqslant m:|| x_{n}\left|b_{n, k}-l\right| \geqslant \epsilon\right\}\right|=0
$$

Consider

$$
\begin{aligned}
\frac{1}{m} \mid\left\{n, k, n^{\prime} \leqslant m\right. & \left.:|| x_{n}\left|b_{n, k}-\right| x_{n^{\prime}(r)}\left|b_{n^{\prime}(r), k}\right| \geqslant \epsilon\right\} \mid \\
& =\frac{1}{m}\left|\left\{n, k, n^{\prime} \leqslant m:|| x_{n}\left|b_{n, k}-l+l-\right| x_{n^{\prime}(r)}\left|b_{n^{\prime}(r), k}\right| \geqslant \epsilon\right\}\right| \\
& =\frac{1}{m}\left|\left\{n, k, n^{\prime} \leqslant m:\left|\left(\left|x_{n}\right| b_{n, k}-l\right)-\left(\left|x_{n^{\prime}(r)}\right| b_{n^{\prime}(r), k}-l\right)\right| \geqslant \epsilon\right\}\right| \\
& \leqslant \max \left\{\frac{1}{m}\left|\left\{n, k \leqslant m:|| x_{n}\left|b_{n, k}-l\right| \geqslant \epsilon\right\}\right|,\right. \\
& \leqslant \max \left\{0, \frac{1}{m}\left|\left\{n^{\prime}, k \leqslant m:|| x_{n^{\prime}(r)}\left|b_{n^{\prime}(r), k}-l\right| \geqslant \epsilon\right\}\right|\right\}
\end{aligned}
$$

Since it is given that $\left(x_{n^{\prime}(r)}\right)$ is convergent to $l$, by Theorem $2.1\left(x_{n^{\prime}(r)}\right)$ is statistically convergent to $l$. That is,

$$
\lim _{m \rightarrow \infty} \frac{1}{m}\left|\left\{n^{\prime}, k \leqslant m:|| x_{n^{\prime}(r)}\left|b_{n^{\prime}(r), k}-l\right| \geqslant \epsilon\right\}\right|=0 .
$$

Now using (2.6) in (2.5) we get,

$$
\lim _{m \rightarrow \infty} \frac{1}{m}\left|\left\{n, k, n^{\prime} \leqslant m:|| x_{n}\left|b_{n, k}-\right| x_{n^{\prime}(r)}\left|b_{n^{\prime}(r), k}\right| \geqslant \epsilon\right\}\right|=0 .
$$

Conversely, let us assume that

$$
\lim _{m \rightarrow \infty} \frac{1}{m}\left|\left\{n, k, n^{\prime} \leqslant m:|| x_{n}\left|b_{n, k}-\right| x_{n^{\prime}(r)}\left|b_{n^{\prime}(r), k}\right| \geqslant \epsilon\right\}\right|=0 .
$$

To prove $\left(x_{n}\right)$ is statistically convergent to $l$, consider

$$
\begin{aligned}
& \frac{1}{m}\left|\left\{n, k \leqslant m:|| x_{n}\left|b_{n, k}-l\right| \geqslant \epsilon\right\}\right| \\
& =\frac{1}{m}\left|\left\{n, k, n^{\prime} \leqslant m:|| x_{n}\left|b_{n, k}-\right| x_{n^{\prime}(r)}\left|b_{n^{\prime}(r), k}+\right| x_{n^{\prime}(r)}\left|b_{n^{\prime}(r), k}-l\right| \geqslant \epsilon\right\}\right| \\
& \leqslant \max \left\{\frac{1}{m}\left|\left\{n, k, n^{\prime} \leqslant m:|| x_{n}\left|b_{n, k}-\right| x_{n^{\prime}(r)}\left|b_{n^{\prime}(r), k}\right| \geqslant \epsilon\right\}\right|\right. \\
& \left.\quad \frac{1}{m}\left|\left\{n^{\prime}, k \leqslant m:|| x_{n^{\prime}(r)}\left|b_{n^{\prime}(r), k}-l\right| \geqslant \epsilon\right\}\right|\right\} \rightarrow 0 \text { as } \quad m \rightarrow \infty, \quad \text { (by (2.6) and (2.7)). }
\end{aligned}
$$

Thus, $\lim _{m \rightarrow \infty} \frac{1}{m}\left|\left\{n, k \leqslant m:|| x_{n}\left|b_{n, k}-l\right| \geqslant \epsilon\right\}\right|=0$. That is, $\left(x_{n}\right)$ is statistically convergent. 
Theorem 2.4. Let $\mathrm{x}=\left(\mathrm{x}_{\mathrm{n}}\right)$ and $\mathrm{y}=\left(\mathrm{y}_{\mathrm{n}}\right)$ be sequences of a non-archimedean Köthe space $\mathrm{K}(\mathrm{B})$. If $\left(\mathrm{x}_{\mathrm{n}}\right)$ is convergent to $l$ and $\left(\mathrm{y}_{\mathrm{n}}\right)$ is statistically convergent to 0 , then $\left(\mathrm{x}_{\mathrm{n}}+\mathrm{y}_{\mathrm{n}}\right)$ is statistically convergent to $l$. i.e., if $x_{n} \rightarrow l(K(B))$ and $y_{n} \rightarrow 0\{S(K(B))\}$, then $\left(x_{n}+y_{n}\right) \rightarrow l\{S(K(B))\}$.

Proof.

$$
\begin{aligned}
& \text { Since } x_{n} \rightarrow l(K(B)), \text { by Theorem 2.1, } \\
& x_{n} \rightarrow l\{S(K(B))\} \\
& \Longrightarrow \lim _{m \rightarrow \infty} \frac{1}{m}\left|\left\{n, k \leqslant m:|| x_{n}\left|b_{n, k}-l\right| \geqslant \epsilon\right\}\right|=0 .
\end{aligned}
$$

Also given,

$$
\begin{gathered}
y_{n} \rightarrow 0\{S(K(B))\} \\
\Longrightarrow \lim _{m \rightarrow \infty} \frac{1}{m}\left|\left\{n, k \leqslant m:|| y_{n}\left|b_{n, k}-0\right| \geqslant \epsilon\right\}\right|=0 . \\
\text { i.e., } \lim _{m \rightarrow \infty} \frac{1}{m}\left|\left\{n, k \leqslant m:|| y_{n}\left|b_{n, k}\right| \geqslant \epsilon\right\}\right|=0 .
\end{gathered}
$$

To prove $\left(x_{n}+y_{n}\right) \rightarrow l\{S(K(B))\}$, i.e., to prove $\lim _{m \rightarrow \infty} \frac{1}{m}\left|\left\{n, k \leqslant m:|| x_{n}+y_{n}\left|b_{n, k}-l\right| \geqslant \epsilon\right\}\right|=0$, consider

$$
\begin{aligned}
& \frac{1}{m}\left|\left\{n, k \leqslant m:|| x_{n}+y_{n}\left|b_{n, k}-l\right| \geqslant \epsilon\right\}\right| \\
& =\frac{1}{m}\left|\left\{n, k \leqslant m:|| x_{n}\left|b_{n, k}-l+\right| y_{n}\left|b_{n, k}\right| \geqslant \epsilon\right\}\right| \\
& \leqslant \max \left\{\frac{1}{m}\left|\left\{n, k \leqslant m:|| x_{n}\left|b_{n, k}-l\right| \geqslant \epsilon\right\}\right|\right. \\
& \left.\frac{1}{m}\left|\left\{n, k \leqslant m:|| y_{n}\left|b_{n, k}\right| \geqslant \epsilon\right\}\right|\right\} \rightarrow 0 \text { as } m \rightarrow \infty, \quad \text { (by (2.8) and (2.9)). }
\end{aligned}
$$

Therefore,

$$
\lim _{m \rightarrow \infty} \frac{1}{m}\left|\left\{n, k \leqslant m:|| x_{n}+y_{n}\left|b_{n, k}-l\right| \geqslant \epsilon\right\}\right|=0
$$

That is, $\left(x_{n}+y_{n}\right) \rightarrow l\{S(K(B))\}$.

\section{Acknowledgment}

My sincere thanks to my supervisor Prof. Srinivasan Vaithinathasamy for his exceptional support, without which this research paper would not have been possible. His knowledge and patience inspired me from the start to the final draft of this paper.

\section{References}

[1] G. Bachman, Introduction to p-Adic Numbers and Valuation Theory, Academic Press, New York-London, (1964).

[2] H. Çakalli, A Study on statistical convergence, Funct. Anal. Approx. Comput., 1 (2009), 19-24.

[3] N. De Grande-De Kimpe, Non-archimedean nuclearity, Study group on ultrametric analysis (Inst. Henri Poincaré), 3 (1982), 8 pages. 1.1

[4] H. Fast, Sur la convergence statistique, (French) Colloq. Math., 2 (1951), 241-244.

[5] J. A. Fridy, On statistical convergence, Analysis, 5 (1985), 301-314. 
[6] J. A. Fridy, H. I. Miller, A matrix characterization of statistical convergence, Analysis, 11 (1991), 59-66.

[7] G. H. Hardy, Divergent Series, Oxford, at the Clarendon Press, Oxford, (1949).

[8] A. F. Monna, Sur le theoreme de Banach-Steinhaus, Nederl. Akad. Wetensch. Proc. Ser. A $66=$ Indag. Math., 25 (1963), 121-131.

[9] L. Narici, E. Beckenstein, G. Bachman, Functional Analysis and Valuation Theory, Marcel Dekker, New York, (1971).

[10] T. Salát, On statistically convergent sequences of real numbers, Math. Slovaca, 30 (1980), 139-150.

[11] W. Śliwa, Every non-normable non-archimedean Köthe space has a quotient without the bounded approximation property, Indag. Mathem. (N.S.), 15 (2004), 579-587. 1.1

[12] K. Suja, V. Srinivasan, On statistically convergent and statistically cauchy sequences in non-archimedean fields, J. Adv. Math., 6 (2014), 1038-1043. 1.3

[13] J. Uma, V. Srinivasan, Statistical convergence in generalized difference sequence spaces over non-archimedean fields, Int. J. Pure Appl. Math., 113 (2017), 280-289. 